\title{
Daily Life Coping with Mucopolysaccharidoses: A Few Devices Showing the Possibilities of Helping Morquio Patients
}

\author{
${ }^{1}$ Basic Music Skills Teacher, Cham, Switzerland \\ 2 Private Dentist, Cham, Switzerland \\ ${ }^{3}$ Construction Apprentice, Cham, Switzerland \\ J Child Sci 2018;8:e144-e150.
}

Christine Wiesbauer Widmer ${ }^{1}$ Alfred Wiesbauer ${ }^{2}$ Sophie Wiesbauer ${ }^{3}$

Address for correspondence Alfred Wiesbauer, MPH, Knonauerstrasse 29, CH 6330 Cham, Switzerland (e-mail: office@wiesbauer.ch).

\author{
Abstract \\ Keywords \\ - mucopoly- \\ saccharidoses \\ - Morquio disease \\ - daily life \\ - spectrum of devices
}

\begin{abstract}
Mucopolysaccharidoses (MPS) show a strong involvement of the skeleton and the joints and partly a significant reduction in growth. Nevertheless, adult patients are particularly interested in being recognized as integrated members of society, and having families and professions. Because of their physical limitations, they always reach their limits. In recent years, numerous devices have come on the market and/or have been improved, which can make everyday life easier. Various improvements are due to new materials such as carbon or silicon and due to electronics/robotics. Amenities such as automated doors, motion detectors, lifts, smartphones with integrated remote control, among others, originally invented for normal consumers prove to be a great help for handicapped persons. What was meant for the masses proves to be extremely useful for patients with MPS.
\end{abstract}

\section{Introduction}

Mucopolysaccharidoses (MPS) belong to the group of lysosomal storage diseases, and are diseases of the extracellular matrix of the connective tissue. They show a strong involvement of the skeleton also known as dysostosis multiplex. ${ }^{1}$ This leads to a significant decrease in growth in some patients, especially for those with Morquio disease. ${ }^{2}$ The fibrous structures of the joints are also involved in all patients, which lead to contractures and reduced mobility in most of the patients with MPS. ${ }^{3}$ However, for those affected by MPS IV, Morquio disease, the clinical symptoms show a high degree of instability of the joints, so that many challenges of everyday life can only be overcome with difficulty. Nevertheless, adult patients are particularly interested in being recognized as integrated members of the society, and want to have families and useful professions.

received

October 28, 2017 accepted after revision June 18, 2018
Issue Theme Advances in mucopolysaccharidoses; Guest Editors: Susanne G. Kircher, MD, PhD, MBA, Adriana M. Montaño, PhD, Christina Lampe, MD.
Because of their physical limitations, they almost always exceed their limits.

In recent years, numerous devices have come to market and/or have been improved. Various improvements are due to new materials such as carbon or silicon and due to electronics/robotics. Amenities such as automated doors, motion detectors, lifts, and smartphones with integrated remote control originally designed for normal consumers prove to be a great help for handicapped persons. What was meant for the masses proves to be extremely useful for this special case. Many items are applicable to MPS IV patients, but they often have to be revised and adapted. These adjustments are expensive, costly, and are often made by interested professionals or inventive relatives of a single patient. How a satisfactory daily life with various devices is possible can be shown by an example of a patient suffering from MPS IVA.

\section{Copyright @ 2018 Georg Thieme Verlag KG Stuttgart · New York}

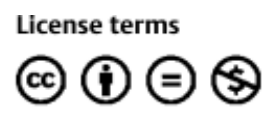




\section{Clinical Presentation of Mucopolysaccharidosis Type IV}

Mucopolysaccharidosis type IV was first described in 1929 by Brailsford and Morquio. ${ }^{4,5}$ It is caused by a disturbance in the dismantling of keratan sulfate, which is predominantly present in the skeleton. Affected persons have a delayed growth with disproportionately normal growing limbs, flattened vertebrae, scoliosis, pectus carinatum, flattened femur heads, and poorly shaped hip joint pans. Due to a slackness of tendons and ligaments, knocked knees, flat feet, movable hand, and finger joints are common. ${ }^{6}$ Particularly dramatic is the instability of the cervical spine, which may be caused by not fully formed structures of the dens axis, and a weakness of the fixing holding apparatus. A possible consequence is compression of the myelon and this may lead to a high-level paraplegia. $^{7}$

Whereas in the first decades patients could only be diagnosed by the increased excretion of keratan sulfate, in 1978 a defective or lacking enzyme was recognized as the cause. In case of Morquio A it is the enzyme $\mathrm{N}$-acetylgalactosamine-6-sulfate-sulfatase (GALNS) and for Morquio B the enzyme $\beta$-galactosidase. The causing genes are the GALNSgene for MPS IVA on chromosome 16q24.3 and the GLB1gene for MPS IVB on chromosome 3p21.33.8,9 An enzyme replacement therapy (Vimizin) is available on the market since 2014 for MPS IVA. ${ }^{10}$

\section{What Are the Main Handicaps in Daily Life?}

Affected persons with Morquio are primarily physically restricted. Their intelligence ranges from normal to above-average. They are active members in society, attending schools, completing education, studying, wishing to be mobile, fostering friendships and partnerships. In the past, many assisting devices already available were adapted for their size, but still they did not provide enough mobility. They were too heavy, too large, too inflexible and mainly served the purpose of relieving caregivers. This is often seen today in patients coming from less developed countries. They sit in oversized, rigid adult wheelchairs or in baby carriages in which they cannot move themselves. These devices do not prevent pathological movements or positions. Wheelchairs can serve for many purposes, that is, they serve as a chair to eat or work at an average table.

Until a few years ago, such experiences were common in the industrialized countries, too. It was almost impossible or only with extraordinary efforts for patients to participate in social life, to pursue a professional life, to practice sports, and to cultivate hobbies.

\section{Spectrum of Devices}

Each device should not be considered on its own, because they are multifunctional and have to meet a variety of requirements, which means that multidisciplinary handicapped persons should be supported in a wide range of activities. The following paragraphs present the experiences we as caring parents have made over the years with such devices. Some tricks are shown, tips are given and we try to explain the advantages as well as the disadvantages of the shown items.

\section{Wheelchairs}

For a long time, children's wheelchairs had to meet the same safety criteria as the wheelchairs for adults (-Fig. 1). They were built to support an average adult and were, therefore, heavy. A child weighing $12 \mathrm{~kg}$ had to move a wheelchair that weighed over $20 \mathrm{~kg}$. For a few years now, the wheelchairs for kids may be built lighter because the regulatory bodies have allowed it. A compromise needs to be found between easy maneuvering and safety especially when getting in and out of the wheelchair because it could tilt due to the change in the center of gravity. The wheels are mounted at a steeper angle, which makes the wheelchair more maneuverable. In general, patients are seated lower to make driving easier and faster. The obvious disadvantage is that normal work tables are then too high. Either the table needs to be adjusted to make life less difficult, or a children's high stool (Tripp Trapp, www. stokke.com) is used. There are many affordable ones available at the small children's shops. As adults, MPS IV patients continue to rely on them because they usually do not grow significantly. They are, therefore, often perceived and treated as small children by society. Therefore, it is important to pay attention to a neutral design.

\section{Power Wheelchairs}

Wheelchairs are called "Freedom Machines." Thanks to the rapid development in the field of electro-mechanics and electronics, Morquio patients have many areas of everyday life in which they can move independently and interact with their environment.

Whether a power wheelchair has a front wheel, a rear wheel or a central drive influences the driving behavior and the well-being of the patients. Some Morquio patients have problems with dizziness or have an unstable cervical spine. Depending on the maneuverability and torque, these problems can be accentuated. An extensive test drive helps to find the best suited model.

The suspension is of central importance. It must be finely adjustable and have a degree of rigidity that does not create

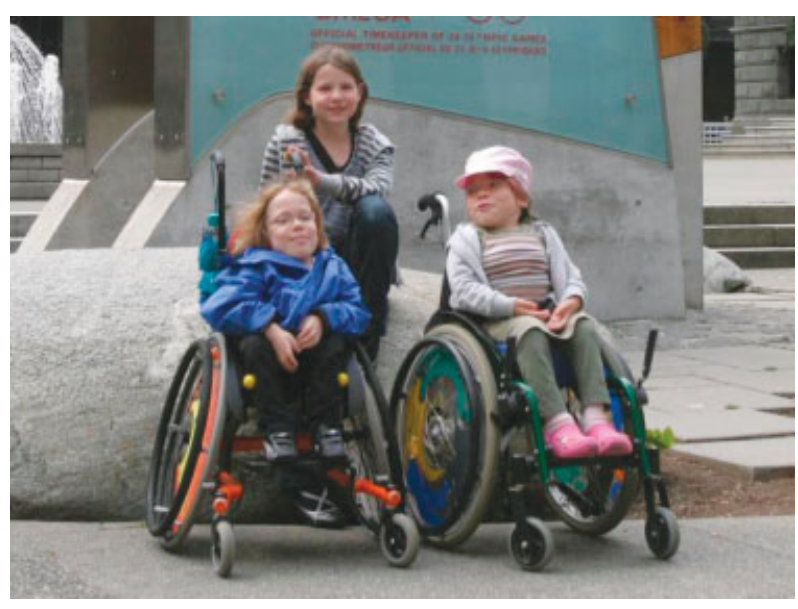

Fig. 1 Wheelchair for small adolescents and adults. 
swings and avoids dizziness. Individual models have a slope and tilt compensation, so depending on the slope of the roadway, the seat aligns and the patient needs to fight less against gravity. This is important for Morquio patients because the head is relatively heavy compared with the body. Together with the instability of the cervical spine and the laxness of all joints, this causes strain and effort.

The seat should always be individually adjusted. Unfortunately, we see all too often insufficient adjustments or standard supplies that harm or do not serve the patient. Depending on the mobility and activity, the seat must be supportive, padded, and allow a defined degree of movement which the patient needs. The seating habits change during the day. In addition, adjustment of the tilt, the backrest, and the armrests is advantageous. If the components are electric, the patient can adjust them independently with the joystick. The armrests deserve special attention. Pressure points on the elbow, especially by incorrectly positioned seams, are to be avoided. The armrests should move so that the operator can always reach the joystick. Otherwise immobile MPS IV patients cannot move out of a reclining position.

Even in an electric wheelchair, a Morquio patient cannot meet others at eye level. A power seat lift is an expensive remedy. The entire seat is raised up to $40 \mathrm{~cm}$. In such a position, the patient does not have to tilt the head backward. This is useful not only in conversations, but also operating light switches and elevator controls can be reached or information boards read in public. ATMs are almost always too high, though. ${ }^{11,12}$

Patients who are still ambulatory must be able to get out of the wheelchair. Because of the small size of the body and the height in which they are sitting, this is a dangerous, strenuous endeavor. Falls and sprains are to be avoided for Morquio patients because of the unstable joints and the spine. The footrest can be equipped with a lift, which brings the standing person to the vicinity of the seat. However, many patients lack the patience to wait long enough for the lift to be in the right position. Another option is to install one or two additional foot rests that are arranged like a staircase. If possible, they should be foldable. The patient must be able to hold on to the chair which also requires adjustment. There is a wheelchair model which brings the entire seat close to the floor like a forklift. This is a good option in the case of immobile toddlers, because they like to play on the ground. ${ }^{13}$

Morquio patients can also drive a car. There are wheelchairs equipped with anchoring devices. By means of a ramp, the patient drives into the car, anchors the wheelchair, and drives the car with the chairs' joystick. A transfer is no longer necessary. The car needs major adaptation, though. ${ }^{14}$

Even in high-complex wheelchairs with special designs, compromises must be made somewhere. Each adjustment affects the entire construction. For example, if the seat is too forward from the intended zone, it can influence the balance, the suspension, and the driving behavior. Ordering a wheelchair from the catalogue and then, with all the changes parents or patients wish to do, it will lead to an unsatisfactory result. The orthopedic technician and the wheelchair mechanic know this problem. Good cooperation between patients, technicians, doctors, therapists, and caregivers is essential.

\section{Environmental Controls}

A power wheelchair serves as the basis for an environmental control. This is made up of different components. For Morquio patients, it is difficult to reach light switches, latches, lift buttons, or window shades due to their small body size and arms limited range of motion. With an environmental control, many domestic facilities can be operated, usually via the joystick of the wheelchair, electromechanically or electronically. In general, lighting, blinds, doors, windows, heating, telephones, TV, video, and HiFi systems can be controlled.

Let me give an example of what can be achieved with an environmental control system:

Returning home after a school or working day shows how difficult the everyday life of a Morquio patient is. The normal procedures are: Unlock the door, open the door, step in, lock the door, take off the jacket and hang it up, remove the shoes, take care of good lighting conditions, enter different rooms, and do a job. Already the first steps are difficult for MPS IV patients. Opening the zipper of a bag, removing a key, inserting it into the lock, turning it over, and pulling it out again are strenuous for the rather weak hands with unstable wrists at a height above the shoulders, and take accordingly too much time and effort. The head must be tilted backward to see the lock. This movement should be avoided as the cervical spine is either unstable or stiffened after surgery. This position can even be painful. A door handle can usually only be reached at the tip of the toes, but it is usable. Door knobs, however, are a greater obstacle; simultaneous overhead turning and pulling are practically impossible. Pushing a door open is manageable but pulling is troublesome.

The light switch has to be pressed over the head again; this is repeated on and off until the desired room is reached. A patient can hardly crank down the blind if the sun is shining too bright. If it is too warm in the room opening a window is a risky job. It is easy to say that a laptop offers many chances. However, to get it out of its case, to unpack it, to put it on the table, to open it up, to turn it on, and to enter the password are a major undertaking for the small-scale patients. The laptop has roughly the size of their upper body and weighs almost $10 \%$ of their own body weight. Pressing keyboard shortcuts is practically impossible due to limited fine motor skills and small hands.

With an environmental control, the patient is more at ease and less at risk. It is attached to the electric wheelchair and wired so that a constant power supply is provided. In many cases, the smartphone is part of this control and is also positioned and supplied with power. The smartphone is exposed to the weather and must be protected accordingly. The individual components are operated with the joystick of the wheelchair or additional buttons. The situation described above can thus be managed much faster and easier. The patient approaches the front door and triggers the door lock release. The door is opened electromechanically, the patient enters the house, and the door closes automatically. 
The room doors can be easily opened with the wheelchair. A rubber buffer at the foot rest prevents excessive noise and damage. Light switches are operated via the joystick and environmental control, as well as blinds. Unpacking the laptop remains a problem. Thanks to a cloud all relevant data can be accessed. If the patient approaches the computer, the machine detects it and turns itself on. Patient can do a job, like homework, answer mails, or watch movies. By means of environmental control, they can also control TV sets, HiFi equipment, house telephones, or video recorders. ${ }^{15}$

Robotics are now also helping disabled people. An example is a robotic arm which is attached to the wheelchair and is operated via a joystick. It helps the immobile people with weak or incapable hands and arms in everyday life and in everyday work. It lifts heavy objects such as bottles or books, which is very difficult for MPS IV patients. Often, they need help in pouring drinks, dressing, combing, crushing food, lifting large, heavy objects, or reaching things that are stored higher up or outside of their arm position. A robotic arm could do this for them. In robotics, there is a wide field to achieve a far greater independence in everyday life, in training, and in professional life. Many models have already made the jump from experimental to market. We can expect more products - Fig. $\mathbf{2}^{16}$

\section{Smartphones}

Smartphones are not only used for communication, although this is their prime intention for Morquio patients as well.

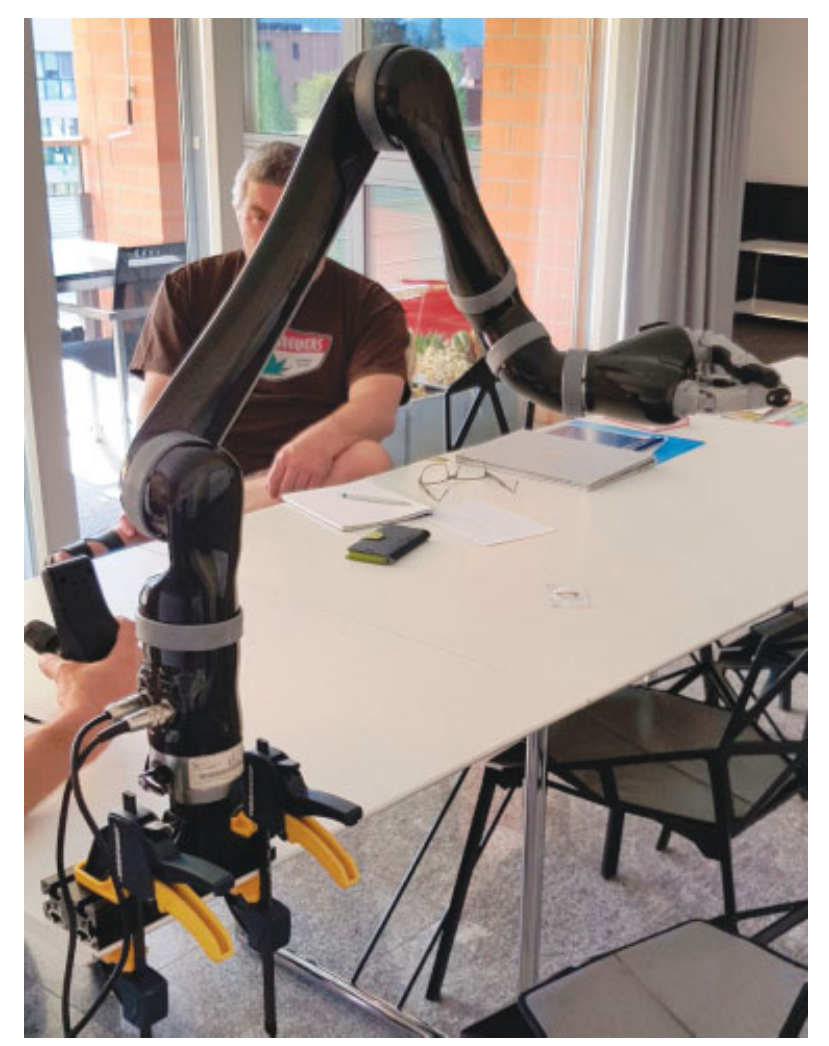

Fig. 2 In case of weak or incapable hands and arms, a robotic arm might be helpful.
They are easy to operate and almost without buttons, but still large for small-scale persons. Holding them to the ear and calling for hours is tedious. It is all the more amazing how long young people do it anyway. Playing games or chatting is also possible with great perseverance. We can take this behavior as a yardstick for those activities in which they tire very quickly.

A modern smartphone can be used as a remote control for the laptop or TV with an appropriate app. It is a device that is handy and always nearby, unlike many remote controls that are often unreachable and have buttons with high resistance.

They even serve as a notebook, homework book, navigation device, and much more. Think of all those media apps! Because there is a plethora of apps available, the right choice is hard to make. Apps that have a pain diary or something like this have proved to be helpful. The smartphone with all its sensors can even be part of the process and all entries are recorded immediately.

MPS patients are often rather deaf and need hearing aids. These small tools are marvels and versatile. Certain models can be connected to smartphones via Bluetooth. They do so with laptops, TV sets, and stereo systems also. With an app, the volume can be adjusted individually. Depending on the environmental situation, other settings are required. In traffic, one wants to hear the approaching vehicles and accepts in case of doubt to miss a conversation. In the classroom, Morquio patients want to understand the classmates, but hide the scratching of the pencil. Patients can even switch to the teacher via a link. The teacher carries a transmitter, the student a receiver and is hearing the voice of the teacher as if wearing headphones. If the teacher does not speak, the ambient sounds are placed in the foreground. In churches or lecture halls, there are such facilities for hearing aid carriers in which they can latch in. Depending on the situation, the patient can select the desired program via the smartphone without having to grab behind the ears and use small buttons or sliders. Lifting the arms is difficult for Morquio patients because the shoulder movement is limited. It is already tricky for the caregivers to operate the small buttons, and it is more difficult for MPS patients. Another Bluetooth device allows the patient to use the hearing aids as headphones. The patient receives a call, hearing the ringing over the hearing aids. He takes the call from the joystick and listens to the caller directly via the hearing aids - Fig. 3. $^{17}$

\section{Electronics}

Laptops are important equipment for MPS patients. They are easy to use by the patients themselves. Most of them are equipped with a camera and provide "ease of use" adjustment options. There are small versions available so that Morquio patients can look over the screen, for example, to follow the instructor or to look at the school blackboard.

With face recognition, logging in is fast and secure. An external touchpad simplifies the operation, because it can be placed optimally. The programming of a special screen keyboard with abbreviations to avoid combinations such as 


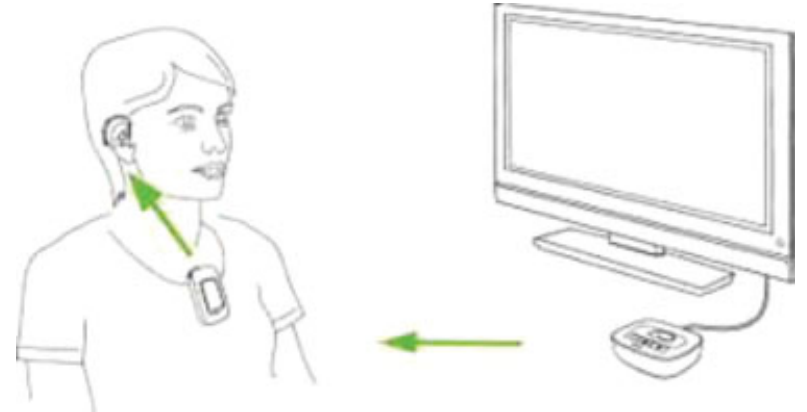

Fig. 3 Patient takes a call from the joystick and listens to the caller directly via the hearing aids.

"control, ALT, delete" is not a problem for savvy laymen anymore. For example, the binomial formulas can be seen in a word prediction on the smartphone. Many patients, caretakers and relatives, however, have other obligations and leave it to professionals from the IT industry. Such a keyboard is important in school, for example. There are screen calculators and calculator apps that already have the desired abbreviations, such as "«shift, = or shift." However, a calculator will solve the equation, thereby minimizing the learning effects. A keyboard only offers to display the character without a keyboard shortcut.

An interesting side effect to notice: Because Morquio patients seem so small and fragile, caregivers are inclined to take over many chores. Morquio patients are very adept in social matters and sympathetic by their wit. Too often, caregivers fall on their tricks and become overprotective. A computer is, however, resistant. ${ }^{18}$

\section{Textbooks Are Either Digitally Available or Can Be Scanned}

Without need to carry heavy books around, the student can devote himself to reading and learning without effort. All worksheets are nowadays created digitally. The teacher emails the page to the student, who can edit it with Word or Acrobat Pro. If required, the sheet is then printed and handed to the teacher, else it is mailed back.

Longer texts such as essays can be written with a dictation program. However, this is not as easy as it sounds. While dictating, the entire text and grammar of the sentence to be dictated have to be ready together. Here, different brain areas work than in normal writing. One can try to write a larger text in this way by predicting and writing the sentence, to later notice that it is incoherent and sounds bumpy. The program is slower than thinking, it slows down the writing flow.

Corrections are not made on an ongoing basis, but only when the sentence is read again. The program does not always understand everything right away, but is capable of learning. It takes time to establish patient's own recognition profile and to continuously save corrections. A major problem is the pitch of the voice, it recognizes above all deep male voices. The voice of a Morquio patient does not correspond to this pitch.
In some areas, using a dictation program is a very good method of writing. This is the case in the school for gap texts, copying or standard letters and translations. Dictating needs to be learned. Patients can practice it and have success with it. However, it takes time initially, which seems to be a contradiction, because Morquio patients want to save time. Another problem is spelling, which is taken over by the program. A great benefit is the command input for formatting. With the help of commands such as "Mark" or similar, it is much easier to format. ${ }^{19}$

Another way to increase the writing speed is to write with word prediction. The AutoCorrect feature also helps to write faster. Case sensitivity, for example, which requires keyboard shortcuts, is done automatically. The clever Morquio children use these also in foreign language teaching and leave the correct writing of the vocabulary to the computer. Often the border between usefulness and too much help is blurred.

Learning vocabulary can also be done much more efficiently through different apps. The vocabulary is written on digital cards. The app or program handles the shuffling and flipping of the cards. These programs know how well the subject memorized a particular word already, so the vocabulary is queried accordingly. It is also possible to take an exam or to learn playfully with a partner. ${ }^{20,21}$

If you look at the satchels of some students, you can ask yourself whether they have strapped the whole desk to their back. If someone is just a meter tall, this is even more absurd. A laptop fits the entire school material and does not weigh much. It can be carried and stowed by most Morquio patients.

In a laptop or tablet, entire movies, music albums, or books can be saved and stored. This makes everyday life much easier. If the patient wants to read a book, he has to go to the bookshelf, take out a book that can only be reached with a ladder, climb on a chair, and then start reading. With an e-book stored on the laptop, everything becomes easier.

Simplification does not always have something to do with spoiling. Basically, the patient should be able to perform all activities without too many tools. However, if a Morquio patient wants to lead a self-determined and self-reliant life, wants to get training in the first job market, and consequently wants to work on the first market, many timeconsuming obstacles must be cleared on this way. Employers expected the same quality as from healthy persons, maybe they tolerate less quantity.

It would be nice if one could acquire 100\% knowledge in $50 \%$ time. Unfortunately, this is pure wishful thinking. This is the price one pays for a varied, interesting life.

\section{Leisure and Sports}

One can deal with electronics for days and be completely satisfied. It is nice, though, to have a hobby that you care about like-minded people. Morquio patients also have interesting hobbies.

Drawing at the computer is a good way to be creative despite the lack of fine motor skills (Microsoft Paint, Photoshop). 


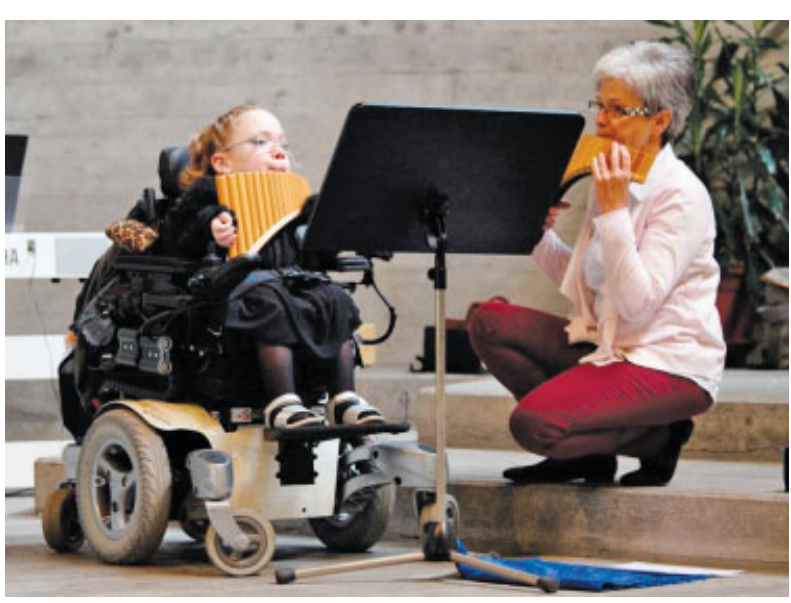

Fig. 4 Patient playing an instrument.

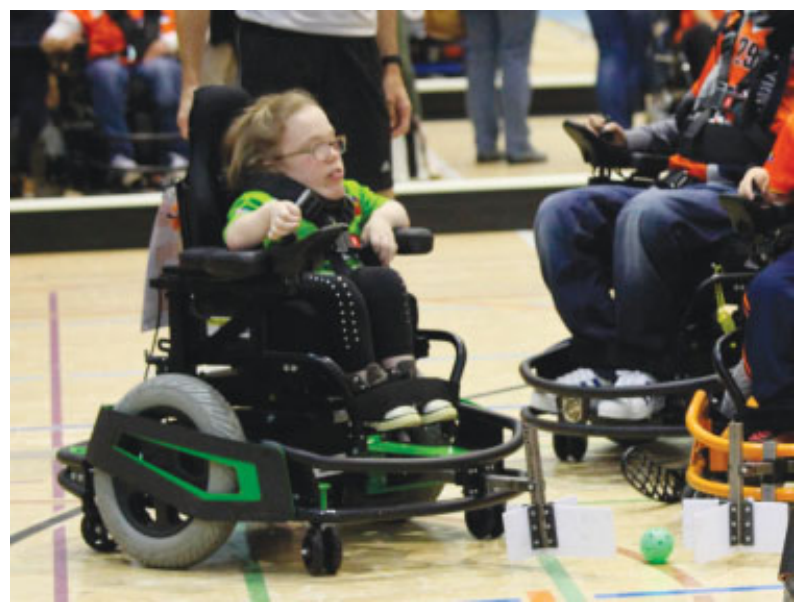

Fig. 5 Team sport with power hockey.

The choice of musical instruments is limited. Usually they are too big, too heavy and concerning fine motor skills are too demanding. Singing is an alternative. Patients can join a choir and enjoy singing together or attending individual lessons (-Fig. 4). Depending on the size and strength of the body, it is possible to play a children's instrument with a limited tonal range. It may sound weaker, yet it is a real instrument with all options. Pan flute does not need fine motor skills, requires a lot of strength and a good lip-stop when blowing. Even a paraplegic Morquio patient has been playing this instrument for years with a lot of fun. 22,23

E-Bikes with support wheels allow the Morquio patient to have outdoor excursions. The support wheels allow the patient to ascend well and drive off. The electric motor supports the forward motion and a brake booster braking. ${ }^{24}$ Alternatively, there are tandem tricycles, where an caretaker takes over the steering and braking. Driving a team sport is a great experience. Here emotions are left free. A good option is the power hockey. This is a sport that is played at club level and in the National League and even internationally (-Fig. 5). ${ }^{25}$

\section{Summary}

Morquio patients usually have a full life ahead of them when the diagnosis is made. Their abilities and inclinations should be stimulated which ensures their good mental health. The aim is to enable the patients to have autonomy and be self-determined in their everyday lives or to be assisted with technical support for as long as possible. Any support of the affected person indirectly relieves the caring relatives, which also helps their mental and physical health. The relationship with friends or partners is facilitated, as they recognize how safely the affected manage everyday life, to the extent that a healthy person cannot imagine.

\section{Conflict of Interest \\ None.}

\section{References}

1 Lachman RS, Burton BK, Clarke LA, et al. Mucopolysaccharidosis IVA (Morquio A syndrome) and VI (Maroteaux-Lamy syndrome): under-recognized and challenging to diagnose. Skeletal Radiol 2014;43(03):359-369

2 Neufeld E, Muenzer J. The mucopolysaccharidoses. In: Scriver CR, Sly WS, Childs B, Beaudet AL, Valle D, Kinzler KW, Vogelstein B, eds. The Metabolic and Molecular Bases of Inherited Disease. 8th ed. New York, NY: McGraw-Hill; 2001:3421-3452

3 Muenzer J. Overview of the mucopolysaccharidoses. Rheumatology (Oxford) 2011;50(Suppl 5):v4-v12

4 Brailsford JF. Chondro-osteo-dystrophy. Roentgenographic and clinical features of a child with dislocation of vertebrae. Am J Surg 1929;7:404

5 Morquio L. Sur une forme de dystrophie osseuse familiale. Arch Med Infants 1929;32:129-135

6 Hendriksz CJ, Harmatz P, Beck M, et al. Review of clinical presentation and diagnosis of mucopolysaccharidosis IVA. Mol Genet Metab 2013;110(1-2):54-64

7 Stevens JM, Kendall BE, Crockard HA, Ransford A. The odontoid process in Morquio-Brailsford's disease. The effects of occipitocervical fusion. J Bone Joint Surg Br 1991;73(05): 851-858

8 Morris CP, Guo XH, Apostolou S, Hopwood JJ, Scott HS. Morquio A syndrome: cloning, sequence, and structure of the human $\mathrm{N}$ acetylgalactosamine 6-sulfatase (GALNS) gene. Genomics 1994; 22(03):652-654

9 Santamaria R, Chabás A, Coll MJ, Miranda CS, Vilageliu L, Grinberg D. Twenty-one novel mutations in the GLB1 gene identified in a large group of GM1-gangliosidosis and Morquio B patients: possible common origin for the prevalent p.R59H mutation among gypsies. Hum Mutat 2006; 27(10):1060

10 Hendriksz CJ. Elosulfase alfa (BMN 110) for the treatment of mucopolysaccharidosis IVA (Morquio A syndrome). Expert Rev Clin Pharmacol 2016;9(12):1521-1532

11 Examples of electric wheelchairs optimized for children. Available at: www.turbo-twist.ch. Accessed January 2018

12 New Zipple Voyage. Available at: www.sunrisemedical.com(Jive). Accessed January 2018

13 SmartDrive MX2+. Stay Independent. Available at: www.permobil.com (K450 MX). Accessed January 2018

14 Examples of electric wheelchairs optimized for children. Available at: www.permobil.com (Permolock). Accessed January 2018

15 Housemate. The Smart Way to Control Your World. Available at: http://housemate.ie. Accessed January 2018 
e150 Daily Life Coping with Mucopolysaccharidoses Widmer et al.

16 Kinova. Achieve Your Best. Available at: www.kinovarobotics. com. Accessed January 2018

17 Phonak. Committed to Changing Lives. www.phonak.com. Accessed January 2018

18 MULTiTEXT für Menschen mit Behinderung. Available at: http:// hindelang-software.de. (Multitext). Accessed January 2018

19 Erstklassige Dokumentationen für den Erfolg Ihres Unternehmens. Available at: www.nuance.de (Dragon naturally speaking). Accessed January 2018

20 Simple Tools for Learning Anything. Available at: https://quizlet. com. Accessed January 2018
21 Die beste App für bessere Noten. Available at: https://www. phase-6.de. Accessed January 2018

22 Nuvo. Available at: www.nuvo-instruments.infowww.kinderinstrumente.ch. Accessed January 2018

23 DAJOERI Panfloeten. Available at: http://panfloeten.ch/. Accessed January 2018

24 Dreiräder für Erwachsene. Available at: http://draisin.net. Accessed January 2018

25 Powerchair Hockey. Available at: www.powerchairhockey.spv. chwww.rodtegg.ch/freizeit/lucerne-sharks. Accessed January 2018 